\title{
Green's Function for Static Klein-Gordon Equation Stated on a Rectangular Region and Its Application in Meteorology Data Assimilation
}

\author{
Hao Cheng ${ }^{1,2} \mathbb{D}$, Xiyu Mu $^{3}$, Hua Jiang ${ }^{2}$, Ming Wei ${ }^{1}$ and Guoqing Liu ${ }^{2, *}$ \\ 1 Collaborative Innovation Center on Forecast and Evaluation of Meteorological Disasters, Nanjing University \\ of Information Science \& Technology, No. 219 Ningliu Road, Nanjing 210044, China; \\ chenghao@njtech.edu.cn (H.C.); njueducn@126.com (M.W.) \\ 2 College of Mathematics \& Physics, Nanjing Tech University, No. 30 Puzhu Road(S), Nanjing 211816, China; \\ quantumcat@163.com \\ 3 Jiangsu Institute of Meteorological Sciences, No. 20 Kunlun Road, Nanjing 210009, China; \\ xiyumufish@gmail.com \\ * Correspondence: guoqing@njtech.edu.cn
}

\section{check for}

updates

Citation: Cheng, H.; Mu, X.; Jiang, H.; Wei, M.; Liu, G. Green's Function for Static Klein-Gordon Equation Stated on a Rectangular Region and Its Application in Meteorology Data Assimilation. Atmosphere 2021, 12, 1602. https://doi.org/10.3390/ atmos12121602

Academic Editors: Baojie He, Ayyoob Sharifi, Chi Feng and Jun Yang

Received: 27 October 2021

Accepted: 29 November 2021

Published: 30 November 2021

Publisher's Note: MDPI stays neutral with regard to jurisdictional claims in published maps and institutional affiliations.

Copyright: (c) 2021 by the authors. Licensee MDPI, Basel, Switzerland. This article is an open access article distributed under the terms and conditions of the Creative Commons Attribution (CC BY) license (https:// creativecommons.org/licenses/by/ $4.0 /)$

\begin{abstract}
The meteorology data assimilation applications often encounter variational problems with unknown weights, where the corresponding Euler equation is an elliptic partial differential equation. This research focused on retrieving the weights in remote sensing data assimilation by means of the computer-friendly form of the Green's function obtained by eigenfunction expansion for the boundary value problem of the static Klein-Gordon equation on a rectangular region. With the help of the proposed retrieving method, the assimilation problem of estimating regional precipitation with weather radar and rain-gauge is solved in the Green's function method. Results show that high accuracy of the proposed method makes it a good candidate for data assimilation problems in operational use.
\end{abstract}

Keywords: Green's function; Klein-Gordon equation; rectangle area; uniformly convergence; regional precipitation; data assimilation

\section{Introduction}

In modern numerical weather prediction, data assimilation is an effective method to improve data accuracy by fusing datasets with different precisions. Data assimilation applications often encounter variational problems, where the corresponding Euler equation is an elliptic partial differential equation (PDE), usually a static Klein-Gordon equation (SKGE). In applications, there are two obstacles: unknown boundary conditions, and unknown weights. Such problems of retrieving the unknown boundary condition and weights are generally known as inverse problems. Most previous research on these problems has concentrated on approximating parameter estimation [1-5] to some extent. Wei, et al. (1998, 2003) calculated the weights with the matrix theory and the finite difference method of partial differential equation [6,7].

In solving elliptic equations by Green's functions, Melnikov (2011) constructed the corresponding Green's functions for the SKGE of several unbounded domains into convergent series, which were more suitable for the numerical implementation [8]. Using Fourier transformation, Aseeri et al. (2015) solved the cubic Klein-Gordon equation and examined strong scaling of the code [9]. Vibrating systems examples were described by Klein-Gordon equation and the correspondence between the classical and quantum settings of this equation was discussed in [10]. Muravey (2015) provided explicit formulas for the Green's functions of an elliptic PDE in an infinite strip and a half-plane [11]. However, due to convergence of series, the explicit Green's function on a rectangular region remains unsolved. Cheng, et al. (2017) found the representation in double series of Green's function 
for SKGE on a rectangular region. Although it is uniformly convergent, the huge amount of computing complexity along with the double series hinders the practical application [12].

Needless to say, the retrieval of the unknown weights of an elliptic PDE requires the explicit solution based on the Green's function, which is in the form of an infinite series. In fact, retrieving the weights is more difficult than retrieving the unknown boundary condition, because the weights appear in the expression of Green's function. Considering the approximation calculation, the infinite series of the Green's function must be acquired in a computer-friendly form. This is the motivation of our research.

This current work obtains a new series representation of the Green's function for the SKGE using eigenfunction expansion. The Green's function is decomposed into a singular component and a regular component. The singular component, with a logarithmic singularity, is expressed as an explicit elementary function, which is computer-friendly for Green's function application. The regular component is expressed as an infinite series. The uniform convergence of this infinite series is investigated. With help of the computerfriendly form Green's function, the weights are calculated by comparing the observations in the interior region with the formula solution of Green's function in the corresponding positions. Real precipitation data assimilation results show that Green's function method is a good candidate for data assimilation problems in operational use.

\section{The Data Assimilation and Its Variational Problem}

In weather prediction areas, a particular physical parameter $u_{R}$ is provided from remote observation. The corresponding physical quantity $\widetilde{u}$ retrieved from $u_{R}$ according to the empirical formula is usually with large error. Some high-precision observation $u_{G}$ in the same area can be used to improve the estimation accuracy, but the number of high-precision observations is limited. More reasonable constraints are also needed to improve the estimation accuracy. According to the smoothness and the dependence on the observation, an unknown function $u(x, y)$ is built to satisfy the following functional extremum problem

$$
\iint_{\Omega}\left[\left(\frac{\partial u}{\partial x}\right)^{2}+\left(\frac{\partial u}{\partial y}\right)^{2}+\mu_{R}\left(u-u_{R}\right)^{2}+\mu_{G}\left(u-u_{G}\right)^{2}\right] d x d y=\min
$$

where $\Omega$ stands for a special field, $\mu_{R} \geq 0, \mu_{G} \geq 0$ are weights corresponding to different error items. In addition, the values of $u(x, y)$ on the boundary $\Gamma$ of area $\Omega$ are given by

$$
\left.u(x, y)\right|_{\Gamma}=\phi(x, y)
$$

For this functional extremum problem with the boundary constraint, the corresponding Euler's equation is presented as follows [13,14]:

$$
\frac{\partial^{2} u}{\partial x^{2}}+\frac{\partial^{2} u}{\partial y^{2}}-\mu u=\hat{u},
$$

where $\hat{u}=\mu_{R} u_{R}+\mu_{G} u_{G}, \mu=\mu_{R}+\mu_{G}$. This is a typical Dirichlet problem for an elliptic equation stated on a special region. Theoretically, the elliptic equation can be solved by the Green's function. However, as mentioned before, the weights are usually unknown in real data assimilation problem.

The Green's function of a PDE depends on not only the form of the equation but also the boundary condition. It is important to find an explicit solution of the Green's function [12]. In order to get an analytical expression of the corresponding Green's function, we consider the special situation of a rectangular area. The solution of Equation (3) has area of

$$
\Omega=\{(x, y) \mid 0 \leq x \leq a, 0 \leq y \leq b\} .
$$


For simplicity of discussion, in this paragraph, let $k^{2}=\mu, f=\hat{u}$, we discuss the Klein-Gordon equation as follows.

$$
\left\{\begin{array}{l}
\nabla^{2} u-k^{2} u=f \\
\left.u\right|_{\Gamma}=\phi
\end{array}\right.
$$

As Hilbert has shown, a boundary problem is solvable if a generalized Green's function is introduced. Therefore, we should find the corresponding Green's functioni.e., $G(x, y ; \xi, \eta)$-which satisfies [13]

$$
\left\{\begin{array}{l}
\nabla^{2} G-k^{2} G=\delta(x-\xi) \delta(y-\eta) \\
\left.G\right|_{\Gamma}=0
\end{array}\right.
$$

for any point $(\xi, \eta)$ in domain $\Omega$. Here, $\delta$ is a Dirac function.

On the other hand, if $u(x, y), G(x, y ; \xi, \eta) \in C^{2}(\Omega)$, from the Green's function, we have

$$
\iint_{\Omega}\left[u \nabla^{2} G-G \nabla^{2} u\right] d x d y=\int_{\Gamma}\left(u \frac{\partial G}{\partial \vec{n}}-G \frac{\partial u}{\partial \vec{n}}\right) d s .
$$

where $\vec{n}$ is normal vector of the boundary $\Gamma$. Let $u$ satisfy Equation (4), substituting $u$ into Equation (6) gives

$$
\iint_{\Omega}\left[u \nabla^{2} G-G\left(k^{2} u+f\right)\right] d x d y=\int_{\Gamma}\left(u \frac{\partial G}{\partial \vec{n}}-G \frac{\partial u}{\partial \vec{n}}\right) d s .
$$

Then, for a generalized Green's function satisfying Equation (5), the above equation can be simplified as

$$
\begin{gathered}
\iint_{\Omega}\left[u\left(\nabla^{2} G-k^{2} G\right)-G f\right] d x d y=\iint_{\Omega} u \delta(x-\xi, y-\eta) d x d y-\iint_{\Omega} G f d x d y \\
=\int_{\Gamma}\left(u \frac{\partial G}{\partial \vec{n}}-G \frac{\partial u}{\partial \vec{n}}\right) d s .
\end{gathered}
$$

The solution of Equation (4) therefore becomes

$$
u(\xi, \eta)=\iint_{\Omega} G(x, y ; \xi, \eta) f(x, y) d x d y+\int_{\Gamma} \phi(x, y) \frac{\partial G(x, y ; \xi, \eta)}{\partial \vec{n}} d s
$$

The next key problem is to get the solution of Equation (5), the generalized Green's function $G(x, y ; \xi, \eta)$.

It is well known that non-homogeneous boundary conditions can be transformed into homogeneous boundary conditions. For simplicity, we assume that $\phi(x, y)=0$ here. Then we get

$$
u(\xi, \eta)=\iint_{\Omega} G(x, y ; \xi, \eta) f(x, y) d x d y=\int_{0}^{b} \int_{0}^{a} G(x, y ; \xi, \eta) f(x, y) d x d y
$$

where the Green's function $G(x, y ; \xi, \eta)$, according to the Appendix A, is defined by

$$
(x, y ; \xi, \eta)=\frac{2}{b} \sum_{n=1}^{\infty} g_{n}(x, \xi) \sin v y \sin v \eta, v=\frac{n \pi}{b}
$$

The coefficients $g_{n}(x, \xi)$ for $x<\xi$ are of the form

$$
\begin{aligned}
g_{n}(x, \xi)= & \frac{1}{4 h \sinh h a}[\exp (h(x-\xi-a))-\exp (h(x+\xi-a)) \\
& +\exp (-h(x-\xi-a))-\exp (-h(x+\xi-a))]
\end{aligned}
$$


where $h=\sqrt{v^{2}+k^{2}}$. Substituting (9), (10) into (8) yields

$$
\begin{aligned}
u(\xi, \eta)= & \frac{2}{b} \int_{0}^{b} \int_{0}^{\xi} \sum_{n=1}^{\infty} \frac{\sinh h(\xi-a) \sinh h x}{h \operatorname{sinhh} a} \sin v y \sin v \eta f(x, y) d x d y \\
& +\frac{2}{b} \int_{0}^{b} \int_{\xi}^{a} \sum_{n=1}^{\infty} \frac{\sinh h{ }^{\tau} \sinh h(x-a)}{h \sinh h a} \sin v y \sin v \eta f(x, y) d x d y
\end{aligned}
$$

where $f=\mu_{R} u_{R}+\mu_{G} u_{G}$.

\section{Retrieving the Weights by Formal Solution}

Obviously, formal solution expression (8) contains unknown parameters, $\mu_{R}, \mu_{G}$. The mechanism for retrieving is to minimize the error between the observations and the analytical solution in the observation points. It is well known that the analytical solution can be obtained if the Green's function of the PDE is known. In the previous section, a computer-friendly representation of the Green's function is developed, the approximation calculation of analytical solution can be implemented by directly truncating the infinite series.

As precipitation data $u_{G}$ measured by rain gauge is more accurate, we try to select the weights that satisfy

$$
J\left(\mu_{R}, \mu_{G}\right)=\sum_{(\xi, \eta) \in \Omega_{G}}\left(u(\xi, \eta)-u_{G}(\xi, \eta)\right)^{2}=\text { min. }
$$

where $\Omega_{G}$ represents the rain gauge point set. According to the knowledge of calculus, we compute the partial derivatives as follows

$$
\begin{aligned}
& \frac{\partial J}{\partial \mu_{R}}=2 \sum_{(\xi, \eta) \in \Omega_{G}}\left(u(\xi, \eta)-u_{G}(\xi, \eta)\right) \frac{\partial u(\xi, \eta)}{\partial \mu_{R}} \\
& =2 \sum_{(\xi, \eta) \in \Omega_{G}}\left(u-u_{G}\right)\left[\int_{0}^{a} \int_{0}^{b} G(x, y ; \xi, \eta) \frac{\partial f}{\partial \mu_{R}} d x d y\right. \\
& \left.\quad+\int_{0}^{a} \int_{0}^{b} \frac{\partial G(x, y ; \xi, \eta)}{\partial \mu_{R}} f d x d y\right], \\
& \begin{array}{r}
\frac{\partial J}{\partial \mu_{G}}=2 \sum_{(\xi, \eta) \in \Omega_{G}}\left(u(\xi, \eta)-u_{G}(\xi, \eta)\right) \frac{\partial u(\xi, \eta)}{\partial \mu_{G}} \\
=2 \sum_{(\xi, \eta) \in \Omega_{G}}\left(u-u_{G}\right)\left[\int_{0}^{a} \int_{0}^{b} G(x, y ; \xi, \eta) \frac{\partial f}{\partial \mu_{G}} d x d y\right. \\
\left.+\int_{0}^{a} \int_{0}^{b} \frac{\partial G(x, y ; \xi, \eta)}{\partial \mu_{G}} f d x d y\right]
\end{array}
\end{aligned}
$$

where $\frac{\partial f}{\partial \mu_{R}}=u_{R}, \frac{\partial f}{\partial \mu_{G}}=u_{G}$,

$$
\begin{aligned}
& \frac{\partial G(x, y ; \xi, \eta)}{\partial \mu_{R}}=\frac{2}{b} \sum \frac{\partial g_{n}(x, \xi)}{\partial \mu_{R}} \sin v y \sin v \eta, \\
& \frac{\partial G(x, y ; \xi, \eta)}{\partial \mu_{G}}=\frac{2}{b} \sum \frac{\partial g_{n}(x, \xi)}{\partial \mu_{G}} \sin v y \sin v \eta
\end{aligned}
$$

with

$$
\begin{aligned}
\frac{\partial g_{n}(x, \xi)}{\partial \mu_{R}} & =\frac{\partial g_{n}(x, \xi)}{\partial h} \frac{\partial h}{\partial \mu_{R}}=\frac{\partial g_{n}(x, \xi)}{\partial h} \frac{1}{2 h}, \\
\frac{\partial g_{n}(x, \xi)}{\partial \mu_{G}} & =\frac{\partial g_{n}(x, \xi)}{\partial h} \frac{\partial h}{\partial \mu_{G}}=\frac{\partial g_{n}(x, \xi)}{\partial h} \frac{1}{2 h}, \\
\frac{\partial g_{n}(x, \xi)}{\partial h} & =\frac{\partial}{\partial h}\left(\frac{\sinh h(\xi-a) \sinh h x}{h \sinh h a}\right) \\
& =\frac{[(\xi-a) \cosh h(\xi-a)+x \cosh h x] h \sinh h a}{h^{2} \sinh ^{2} h a} \\
& -\frac{(\sinh h a+h \cosh h a) \sinh h(\xi-a) \sinh h x}{h^{2} \sinh ^{2} h a} .
\end{aligned}
$$




$$
\begin{aligned}
\frac{\partial g_{n}(x, \xi)}{\partial \mu_{R}} & =\frac{\partial g_{n}(x, \xi)}{\partial h} \frac{\partial h}{\partial \mu_{R}}=\frac{\partial g_{n}(x, \xi)}{\partial h} \frac{1}{2 h}, \\
\frac{\partial g_{n}(x, \xi)}{\partial \mu_{G}} & =\frac{\partial g_{n}(x, \xi)}{\partial h} \frac{\partial h}{\partial \mu_{G}}=\frac{\partial g_{n}(x, \xi)}{\partial h} \frac{1}{2 h}, \\
\frac{\partial g_{n}(x, \xi)}{\partial h} & =\frac{\partial}{\partial h}\left(\frac{\sinh h(\xi-a) \sinh h x}{h \sinh h a}\right) \\
& =\frac{[(\xi-a) \cosh h(\xi-a)+x \cosh h x] h \sinh h a}{h^{2} \sinh ^{2} h a} \\
& -\frac{(\sinh h a+h \cosh h a) \sinh ^{2} h(\xi-a) \sinh h x}{h^{2} \sinh ^{2} h a} .
\end{aligned}
$$

Finally, the steepest descent algorithm is designed for solving $J(\vec{z}) \triangleq J\left(\mu_{R}, \mu_{G}\right)=\min$. Algorithm:

Step 1. Choose initial point $\vec{z}^{(0)}=\left(\mu_{R}^{(0)}, \mu_{G}^{(0)}\right), l=0$, precise requirement $\varepsilon>0$;

Step 2. Calculate $\nabla J\left(\vec{z}^{(l)}\right)=\left(\frac{\partial J}{\partial \mu_{R}}, \frac{\partial J}{\partial \mu_{G}}\right) \vec{z}^{(l)}, \vec{z}^{(l)}=\left(\mu_{R}^{(l)}, \mu_{G}^{(l)}\right)$. If $\left\|\nabla J\left(\vec{z}^{(l)}\right)\right\|<\varepsilon$, stop. Otherwise, let $\vec{d}^{(l)}=-\nabla J\left(\vec{z}^{(l)}\right)$;

Step 3. Let $\vec{z}^{(l+1)}=\vec{z}^{(l)}+\lambda_{l} \vec{d}^{(l)}$, find $\lambda_{l}$ from $J\left(\vec{z}^{(l+1)}\right)=\min$;

Step 4. Let $l=l+1$, turn to Step 2 .

According to the Appendix B, convergence analysis and truncation error analysis, the series representation of Green's function is uniformly converged, and the calculation of infinite series can be truncated.

\section{Numerical Experiments}

Simulations are conducted here to find the rate of convergence, and so investigate the performance of the proposed Green's function in Equation (7). Figure 1 plots $G(x, y ; \xi, \eta)$ on the rectangular region $(0 \leq x \leq 80,0 \leq y \leq 60)$ selected from the radar detection range for $(\xi, \eta)$ equals to $(40,30)$. Note that $G$ equals 0 on the boundaries of the region and tends to $-\infty$ when $(x, y)$ tends to $(\xi, \eta)$. The convergence performance of the Green's function on many points in the rectangular area is tested to perform the simulation. Results show that all the points converge steadily to their true numerical values after a limited number of iterations. The convergence speed of the Green's function is much faster when $(x, y)$ is far from $(\xi, \eta)$.

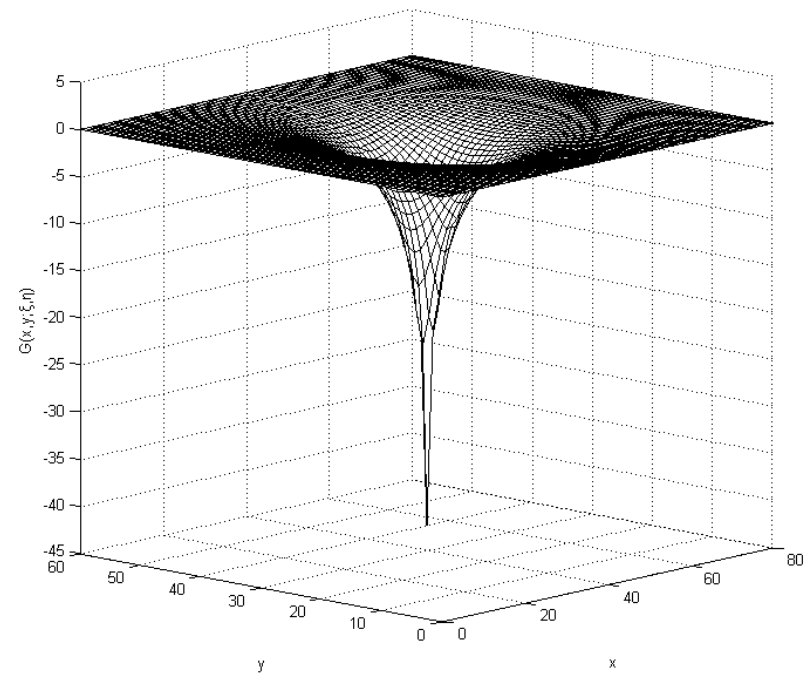

Figure 1. Plot of the Green's function $G(x, y ; \xi, \eta)$ on a rectangular region. 
In the following example, the Green's function method in Equation (8) is used to retrieve the rainfall value at some points in a rectangular area. The results are compared with the values from the Z-I relationship. The observation data are made by Changzhou radar at 18:38 UTC on 11 June 2016 (Figure 2). There are 624 rain gauges in the observation area. In the data assimilation problem, the 624 rain-gauges data are used to correct radarretrieved rainfall. The optimal estimation weights are $\mu_{R}=12, \mu_{G}=96$.

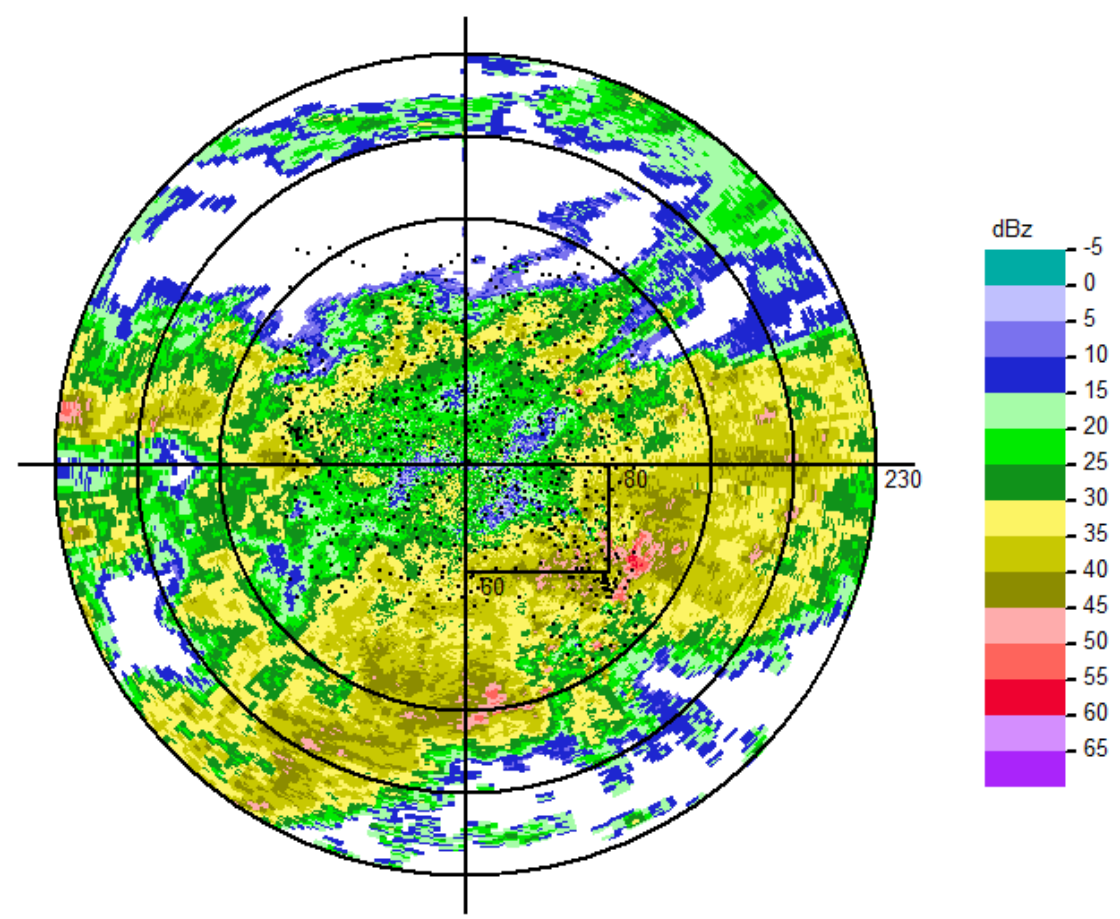

Figure 2. Radar observation and rain-gauge point position.

A rectangular region of $80 \times 60 \mathrm{~km}^{2}$ (Figure 3) and 103 rain gauges inside it are chosen to perform the Green's function method. Because it is a rectangular region, using partial derivative to replace directional derivative, we get the curve integral on the four boundaries by difference method. Assuming boundary condition equals the radar observation, rainfall of every rain-gauge point in the rectangular region is obtained by the Green's function method.

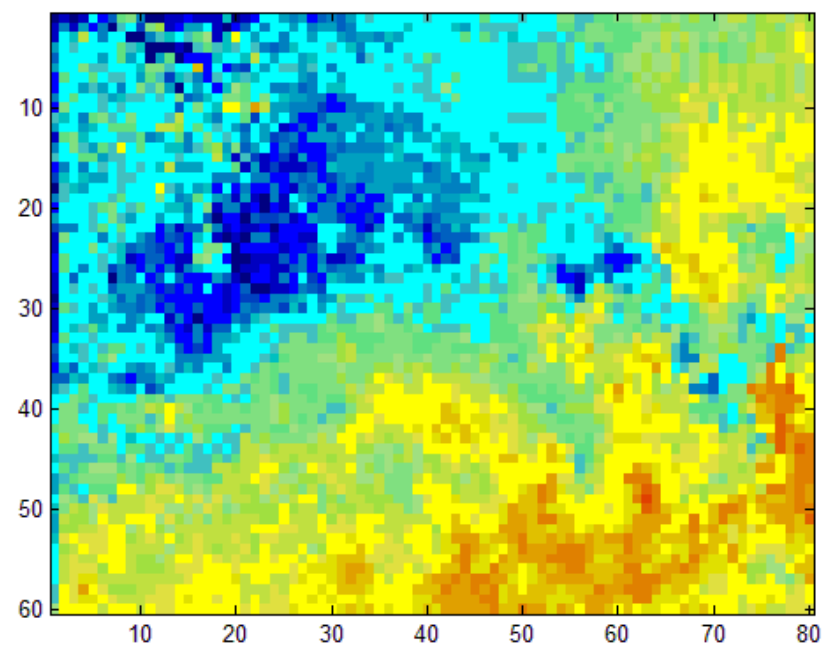

Figure 3. Rectangular Region $\left(80 \times 60 \mathrm{~km}^{2}\right)$. 
In practical application, if the number of rain gauges is insufficient, virtual observations on positions without rain gauges can be obtained by linear interpolation. In our experiment, there are few rain gauges near the bottom of the rectangular area. At $(50,50)$, for example, the observed value 0.953 is obtained by interpolation of five nearby observation points $(42,55),(47,41),(57,46),(50,45)$ and $(53,48)$, which can be used for subsequent parameter estimation or comparative analysis.

In the calculation, 75 rain gauges are selected to estimate the weights, and the remaining quarter of the rain gauges are used for comparative analysis. The results show that the retrieved values possess better correlation properties of the rain-gauge observations than radar observations (Figure 4). Correlation coefficient has grown from 0.6149 to 0.7844 . Most retrieved values of the 103 points are closer to the rain-gauge observations than the values from Z-I relationship of radar and rainfall. Under the condition of optimal weights selection, the rainfall field obtained by variational inversion has higher accuracy. In this case, $\mathrm{Z}$-I relationship is $\mathrm{Z}=296 * \mathrm{I}^{1.24}$ in which $\mathrm{Z}$ is the radar reflectivity, $\mathrm{I}$ is the rainfall $u_{R}$. Table 1 shows some of these values.

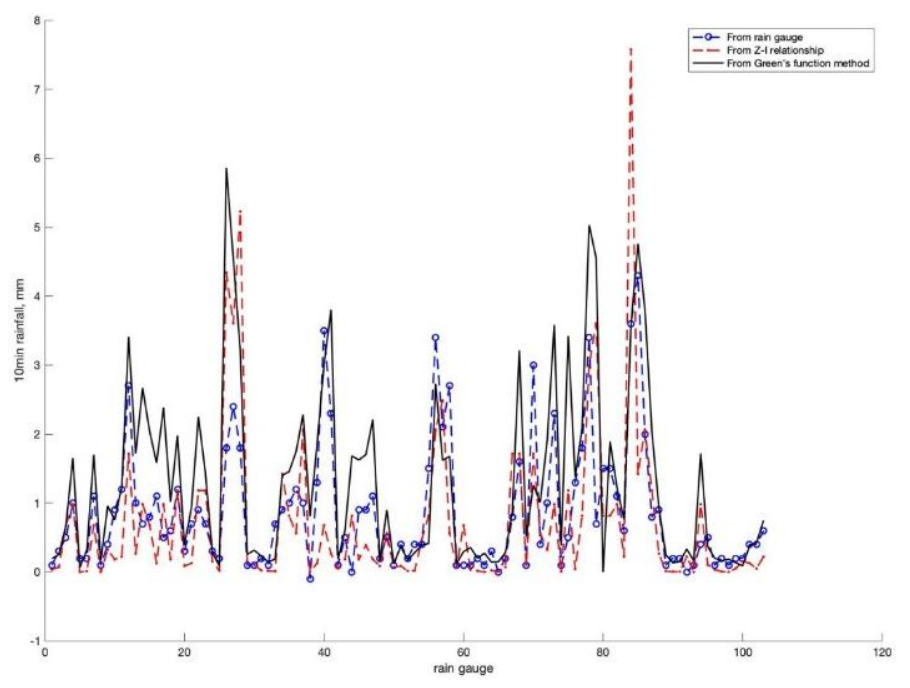

Figure 4. 10 min rainfall from different methods.

Table 1. Interior point values. Pos is interior point coordinates (unit: $\mathrm{km}$ ). Obs is rain-gauge observation in $10 \mathrm{~min}$ (unit: $\mathrm{mm}$ ). Rad is radar observation on the point (unit: $\mathrm{dBz}$ ). Rtr is retrieved data by Z-I relationship of radar and rainfall (unit: $\mathrm{mm}$ ). Joi is the joint value by the Green's function method (unit: $\mathrm{mm}$ ).

\begin{tabular}{ccccccccc}
\hline Pos & $\mathbf{( 1 7 , 4 7 )}$ & $\mathbf{( 5 9 , 2 1 )}$ & $\mathbf{( 6 2 , 5 8 )}$ & $\mathbf{( 7 8 , 3 6 )}$ & $\mathbf{( 6 7 , 4 4 )}$ & $\mathbf{( 5 0 , 2 8 )}$ & $\mathbf{( 2 8 , 2 2 )}$ & $\mathbf{( 5 2 , 2 5 )}$ \\
\hline Obs & 1.2 & 0.7 & 1.8 & 2.7 & 2.3 & 0.9 & 0.2 & 0.6 \\
\hline Rad & 30 & 27 & 47 & 35 & 38 & 31 & 11 & 30 \\
\hline Rtr & 1.2322 & 0.9353 & 3.2013 & 1.6780 & 3.5835 & 0.9404 & 0.1644 & 0.7484 \\
\hline Joi & 0.2224 & 0.1274 & 5.2259 & 0.5629 & 0.9826 & 0.2678 & 0.0065 & 0.2224 \\
\hline
\end{tabular}

\section{Conclusions}

This study solves the data assimilation problems of two kinds of data, $u_{R}$ and $u_{G}$, through a variational model. In order to retrieve the weighting parameters $\mu_{R}, \mu_{G}$ from some of the observations in the interior region, the computer-friendly form of the Green's function is obtained by eigenfunction expansion for the boundary value problem of the static Klein-Gordon equation on a rectangular region. Convergence analysis in Appendix B proves that the series representation of Green's function is computer-friendly, and can be used to approximate computations by a direct truncation. 
The numerical experiment's result shows that the series representation of Green's function converges steadily and rapidly. The Green's function method is used to retrieve the weights and assimilate precipitation data from weather radar and rain-gauges in the experiments. Results show that the Green's function method is a good candidate for data assimilation problems in operational use.

Author Contributions: Conceptualization, H.C., M.W. and G.L.; methodology, H.C. and G.L.; software, H.C. and H.J.; validation, H.C. and X.M.; writing—original draft preparation, H.C. and G.L.; writing-review and editing, H.C. and G.L. All authors have read and agreed to the published version of the manuscript.

Funding: This work was partially supported by National Nature Science Foundation of China (No. 61501224).

Acknowledgments: This work was partially supported by National Nature Science Foundation of China (No. 61501224). We are grateful to anonymous reviewers and an associate editor whose comments led to many improvements of the article.

Conflicts of Interest: The authors declare no conflict of interest.

\section{Appendix A. The Green's Function for the SKGE Stated on a Rectangular Region}

For the elliptic two-dimensional SKGE $\left(\nabla^{2}-k^{2}\right) u(x, y)=f(x, y),(x, y) \in \Omega$, the corresponding homogeneous boundary condition is $\left\{\begin{array}{l}u(x, 0)=u(x, b)=0 \\ u(0, y)=u(a, y)=0\end{array}\right.$. Focusing on the Dirichlet problem set up on a rectangle $\Omega=\{(x, y)\} \mid 0 \leq x \leq a, 0 \leq y \leq b$, we study the following series representation of its Green's function

$$
(x, y ; \xi, \eta)=\frac{2}{b} \sum_{n=1}^{\infty} g_{n}(x, \xi) \sin v y \sin v \eta, v=\frac{n \pi}{b} .
$$

The coefficients $g(x, \xi)$ for $x<\xi$ are of the form

$$
\begin{gathered}
g_{n}(x, \xi)=\frac{1}{4 h \sinh h a} \quad[\exp (h(x-\xi-a))-\exp (h(x+\xi-a)) \\
+\exp (-h(x-\xi-a))-\exp (-h(x+\xi-a))]
\end{gathered}
$$

where $h=\sqrt{v^{2}+k^{2}}$. In fact, it is not difficult to find that

$$
\left.g_{n}(x, \xi)\right|_{x=0}=\frac{\exp (-h(\xi+a))-\exp (h(\xi-a))+\exp (h(\xi+a))-\exp (-h(\xi-a))}{4 h \sinh h a} \neq 0
$$

and $\left.g_{n}(x, \xi)\right|_{x=0} \rightarrow \infty$ as $h \rightarrow \infty$, which yields the non-computer-friendly series representation. However, we find that the correct form is as follows.

$$
\begin{aligned}
g_{n}(x, \xi)=\frac{\sinh h(\xi-a) \sinh h x}{h \sinh h a} & \\
=\frac{1}{4 h \sinh h a} & {[\exp (h(x+\xi-a))-\exp (h(x-\xi+a))} \\
& +\exp (-h(x+\xi-a))-\exp (-h(x-\xi+a))]
\end{aligned}
$$

The following focuses on deducing Equation (A3). Substituting Equation (A1) into the SKGE gives the coefficient function $g_{n}(x, \xi)$ satisfying the differential equation

$$
g_{n}^{\prime \prime}(x, \xi)-\left(v^{2}+k^{2}\right) g_{n}(x, \xi)=\delta(x-\xi)
$$

with homogeneous boundary condition $g_{n}(0, \xi)=0, g_{n}(a, \xi)=0$. For $x$ equal to anything but $\xi, g_{n}^{\prime \prime}(x, \xi)-\left(v^{2}+k^{2}\right) g_{n}(x, \xi)=0$. Therefore,

$$
g_{n}(x, \xi)=\left\{\begin{array}{l}
c_{1} \exp (h x)+c_{2} \exp (-h x), \quad x<\xi \\
d_{1} \exp (h(x-a))+d_{2} \exp (-h(x-a)), x \geq \xi
\end{array}\right.
$$


To determine the above constants $c_{1}, c_{2}, d_{1}, d_{2}$, integrate differential Equation (A4) from $\xi-\varepsilon$ to $\xi+\varepsilon$, where $\varepsilon$ is infinitesimal. The result is $\left.\frac{d g_{n}}{d x}\right|_{\xi-\varepsilon} ^{\xi+\varepsilon}=1$. Integrating again gives $\left.g_{n}\right|_{\tilde{\xi}-\varepsilon} ^{\tilde{\xi}+\varepsilon}=0$. That is, $g_{n}(x, \xi)$ as a function of $x$ is continuous at $x=\xi$, but its first derivative jumps by +1 at that point, leading to

$$
c_{1} \exp (h \xi)+c_{2} \exp (-h \xi)=d_{1} \exp (h(\xi-a))+d_{2} \exp (-h(\xi-a))
$$

and

$$
d_{1} \exp (h(\xi-a))-d_{2} \exp (-h(\xi-a))-\left(c_{1} \exp (h \xi)-c_{2} \exp (-h \xi)\right)=1 / h .
$$

Considering the homogeneous boundary conditions gives $\left\{\begin{array}{l}c_{1}+c_{2}=0 \\ d_{1}+d_{2}=0\end{array}\right.$, from which

$$
\begin{aligned}
c_{1} & =\frac{1}{2 h} \frac{\sinh h(\xi-a)}{\sinh h h^{\tau} \cosh h(\tilde{\xi}-a)-\sinh h(\xi-a) \cosh h \tilde{\xi}} \\
& =\frac{1}{2 h} \frac{\sinh h(\xi-a)}{\sinh h a}
\end{aligned}
$$

and

$$
\begin{aligned}
& d_{1}=\frac{1}{2 h \cosh h(\tilde{\zeta}-a)} \frac{\sinh h(\tilde{\zeta}-a) \cosh h \tilde{\xi}}{\sinh h \zeta^{\tau} \cosh h(\tilde{\zeta}-a)-\sinh h(\xi-a) \cosh h \tilde{\zeta}} \\
& =\frac{\sinh h(\xi-a) \cosh h \tau}{2 h \cosh h(\xi-a) \sinh h a}=\frac{1}{2 h} \frac{\sinh h \xi}{\sinh h a}
\end{aligned}
$$

Hence,

$$
g_{n}(x, \xi)=\left\{\begin{array}{l}
\frac{\sinh h(\xi-a) \sinh h x}{h \sinh h a}, x<\xi \\
\frac{\sinh h \tilde{s} \sinh h(x-a)}{h \sinh h a}, x \geq \xi
\end{array} .\right.
$$

This yields the desired Green's function $G(x, y ; \xi, \eta)$ on the rectangle $\Omega=\{(x, y)\} \mid$ $0 \leq x \leq a, 0 \leq y \leq b$ in the form

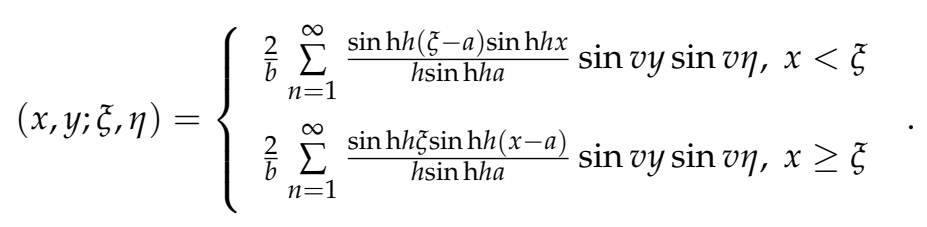

In order to prove the convergence, rewrite the branch of the function $g_{n}(x, \xi)$, which is valid for $x<\xi$, in the form

$$
\begin{aligned}
g_{n}(x, \xi)= & \frac{1}{h} \frac{\sinh h(\xi-a) \sinh h x}{\sinh h a}-\frac{\exp (h(x+\xi-2 a))}{2 h} \\
& \quad+\left[\frac{\exp (h(x+\xi-2 a))}{2 h}-\frac{\exp (v(x+\xi-2 a))}{2 v}\right]+\frac{\exp (v(x+\tilde{\xi}-2 a))}{2 v} \\
= & H_{n}(x, \xi)+M_{n}(x, \xi)+\frac{\exp (v(x+\xi-2 a))}{2 v}
\end{aligned}
$$

where

$$
\begin{aligned}
H_{n}(x, \xi)= & \frac{1}{h} \frac{\sinh h(\xi-a) \sinh h x}{\sinh h a}-\frac{\exp (h(x+\xi-2 a))}{2 h}, \\
& M_{n}(x, \xi)=\frac{\exp (h(x+\xi-2 a))}{2 h}-\frac{\exp (v(x+\xi-2 a))}{2 v} .
\end{aligned}
$$

This lets Equation (A1) be transformed into the following expression

$$
\begin{aligned}
(x, y ; \xi, \eta) & =\frac{2}{b} \sum_{n=1}^{\infty} H_{n}(x, \xi) \sin v y \sin v \eta+\frac{2}{b} \sum_{n=1}^{\infty} M_{n}(x, \xi) \sin v y \sin v \eta \\
& +\frac{1}{b} \sum_{n=1}^{\infty} \frac{\exp (v(x+\xi-2 a))}{v} \sin v y \sin v \eta
\end{aligned}
$$


with

$$
\begin{aligned}
\sum_{n=1}^{\infty} & \frac{\exp (v(x+\xi-2 a))}{v} \sin v y \sin v \eta \\
& =\frac{1}{2} \sum_{n=0}^{\infty} \frac{\exp (v(x+\xi-2 a))}{v} \cos v(y-\eta) \frac{1}{2} \sum_{n=0}^{\infty} \frac{\exp (v(x+\tilde{\zeta}-2 a))}{v} \cos v(y+\eta) \\
& =\frac{1}{4 \pi}\left[\ln \frac{1-2 \exp (c(x+\xi-2 a)) \cos (y+\eta)+\exp (2 c(x+\xi-2 a))}{1-2 \exp (c(x+\xi-2 a)) \cos (y-\eta)+\exp (2 c(x+\xi-2 a))}\right]
\end{aligned}
$$

where $c=\pi / b$. Substituting Equation (A9) into Equation (A8) then gives the new series representation of the Green's function for the Dirichlet problem set up for the SKGE on the rectangle $\Omega$ :

$$
\begin{aligned}
& G(x, y ; \xi, \eta)=\frac{2}{b} \sum_{n=1}^{\infty} H_{n}(x, \xi) \sin v y \sin v \eta+\frac{2}{b} \sum_{n=1}^{\infty} M_{n}(x, \xi) \sin v y \sin v \eta \\
& +\frac{1}{4 \pi}\left[\ln \frac{1-2 \exp (c(x+\xi-2 a)) \cos (y+\eta)+\exp (2 c(x+\xi-2 a))}{1-2 \exp (c(x+\xi-2 a)) \cos (y-\eta)+\exp (2 c(x+\xi-2 a))}\right], x<\xi
\end{aligned}
$$

The representation of $G(x, y ; \xi, \eta)$ valid for $x \geq \xi$ can be derived from Equation (A10), by interchanging the variables $x$ and $\xi$ in the first of the two series only, because the second series is invariant to the swap.

\section{Appendix B. Convergence Analysis and Truncation Error Analysis}

For the effective computational implementation of the representation in Equation (A10), we study the two series separately in the following paragraphs. First, consider the series $\sum_{n=1}^{\infty} H_{n}(x, \xi) \sin v y \sin v \eta$. As it is difficult to prove directly the uniform convergence of the first of the two series in Equation (A10), decompose the series into two parts, one of which is a complete summation. Indeed, the complete summation can remove the disadvantage affecting the uniformly convergent criterion. This is the basic idea of the uniformly convergent proof of the infinite series. Elementary algebra shows

$$
\begin{aligned}
& H_{n}(x, \xi)=\frac{1}{h} \frac{\sinh h(\xi-a) \sinh h x}{\sinh h a}-\frac{\exp (h(x+\xi-2 a))}{2 h} \\
&= \frac{2 \sinh h(\xi-a) \sinh h x-\exp (h(x+\xi-2 a)) \sinh h a}{2 h \sinh h a} \\
&= \frac{1}{4 h \sinh h a}[(\exp (h(\xi-a))-\exp (-h(\xi-a)))(\exp (h x)-\exp (-h x)) \\
& \quad-\exp (-h(x+\xi-2 a))(\exp (h a)-\exp (-h a))] \\
&= \frac{1}{4 h \sinh h a}[-\exp (h(x-\xi+a))-\exp (-h(x-\xi+a)) \\
& \quad\quad \exp (-h(x+\xi-a))+\exp (h(x+\xi-3 a))] \\
&= \frac{1}{2 h(1-\exp (-2 h a))}[-\exp (h(x-\xi))-\exp (-h(x-\xi+2 a)) \\
&\quad+\exp (-h(x+\xi))+\exp (h(x+\xi-4 a))] \\
&=\frac{\exp (-h(x+\xi))-\exp (h(x-\xi))}{2 h(1-\exp (-2 h a))} \\
& \quad+\frac{\exp (h(x+\xi-4 a))-\exp (-h(x-\xi+2 a))}{2 h(1-\exp (-2 h a))} \\
&=\frac{\exp (-h(x+\xi))-\exp (h(x-\xi))}{2 h}+H_{n}^{\prime}(x, \xi)
\end{aligned}
$$

where

$$
\begin{aligned}
H_{n}^{\prime}(x, \xi)=\frac{1}{2 h(1-\exp (-2 h a))} \quad[\exp (h(x+\xi-4 a))-\exp (-h(x-\xi+2 a)) \\
+\exp (-h(x+\xi+2 a))-\exp (h(x-\xi-2 a))]
\end{aligned} .
$$


and

$$
\begin{gathered}
\sum_{n=1}^{\infty} \frac{\exp (-h(x+\xi))-\exp (h(x-\xi))}{2 h} \sin v y \sin v \eta \\
=-\frac{1}{4} \sum_{n=1}^{\infty} \frac{\exp (-h(x+\xi))-\exp (h(x-\xi))}{h}[\cos v(y+\eta)-\cos v(y-\eta)] \\
=\frac{1}{8 c}\left[\ln \frac{1-2 \exp (c(x-\xi)) \cos (y+\eta)+\exp (2 c(x-\xi))}{1-2 \exp (c(x-\xi)) \cos (y-\eta)+\exp (2 c(x-\xi))}\right. \\
\left.\quad-\ln \frac{1-2 \exp (-c(x+\xi)) \cos (y+\eta)+\exp (-2 c(x+\xi))}{1-2 \exp (-c(x+\xi)) \cos (y-\eta)+\exp (-2 c(x+\xi))}\right]
\end{gathered}
$$

Then the Green's function in the form of Equation (A10) can be rewritten as

$$
\begin{array}{r}
(x, y ; \xi, \eta)=\frac{1}{4 \pi} \quad\left[\ln \frac{1-2 \exp (c(x+\xi-2 a)) \cos (y+\eta)+\exp (2 c(x+\xi-2 a))}{1-2 \exp (c(x+\xi-2 a)) \cos (y-\eta)+\exp (2 c(x+\xi-2 a))}\right. \\
+\ln \frac{1-2 \exp (c(x-\xi)) \cos (y+\eta)+\exp (2 c(x-\xi))}{1-2 \exp (c(x-\xi)) \cos (y-\eta)+\exp (2 c(x-\xi))} \\
\left.-\ln \frac{1-2 \exp (-c(x+\xi)) \cos (y+\eta)+\exp (-2 c(x+\xi))}{1-2 \exp (-c(x+\xi)) \cos (y-\eta)+\exp (-2 c(x+\xi))}\right] \\
+\frac{2}{b} \sum_{n=1}^{\infty} H_{n}^{\prime}(x, \xi) \sin v y \sin v \eta+\frac{2}{b} \sum_{n=1}^{\infty} M_{n}(x, \xi) \sin v y \sin v \eta
\end{array}
$$

To explore the convergence of $\sum_{n=1}^{\infty} H_{n}^{\prime}{ }_{n}(x, \xi) \sin v y \sin v \eta$ and analyze its truncation errors requires estimation of the asymptotic property of the common term $H^{\prime}{ }_{n}(x, \xi)$.

As $0 \leq x<\xi \leq a$, we have

$$
\begin{aligned}
&\left|H_{n}^{\prime}(x, \xi)\right|=\mid \frac{1}{2 h(1-\exp (-2 h a))}[\exp (h(x+\xi-4 a))-\exp (-h(x-\xi+2 a)) \\
&+\exp (-h(x+\xi+2 a))-\exp (h(x-\xi-2 a))] \mid \\
&=\mid \frac{\exp (-h a)}{2 h(1-\exp (-2 h a))}[\exp (h(x+\xi-3 a))-\exp (-h(x-\xi+a)) \\
&+\exp (-h(x+\xi+a))-\exp (h(x-\xi-a))] \mid \\
& \leq \frac{\exp (-h a)}{2 h(1-\exp (-2 h a))}[\exp (h(x+\xi-3 a))-\exp (-h(x-\xi+a)) \\
&\quad+\exp (-h(x+\xi+a))-\exp (h(x-\xi-a))] \\
& \leq \frac{2 \exp (-h a)}{h(1-\exp (-2 h a))}=O\left(\frac{1}{h \exp (h a)}\right), h \rightarrow \infty .
\end{aligned}
$$

Hence, $\left|H^{\prime}{ }_{n}(x, \xi) \sin v y \sin v \eta\right| \leq \frac{2 \exp (-h a)}{h(1-\exp (-2 h a))}$. The uniform convergence of the series $\sum_{n=1}^{\infty} \frac{2 \exp (-h a)}{h(1-\exp (-2 h a))}$, from the series convergence criterion, clearly leads to the uniform convergence of the series $\sum_{n=1}^{\infty} H_{n}^{\prime}(x, \xi) \sin v y \sin v \eta$.

In order to compute approximately the Green's function, analyze the modulus of its remainder.

$$
\begin{gathered}
\left|\sum_{n=N+1}^{\infty} H_{n}^{\prime} \sin v y \sin v \eta\right| \leq \sum_{n=N+1}^{\infty} \frac{2}{(1-\exp (-2 h a)) h \exp (h a)} \\
\leq \frac{2}{(N+1)(1-\exp (-2(N+1) a))} \sum_{n=N+1}^{\infty} \frac{1}{\exp (n a)} \\
=\frac{2 \exp (-(N+1) a)}{(N+1)(1-\exp (-2(N+1) a))(1-\exp (-a))}
\end{gathered}
$$

Hence, the series $\sum_{n=1}^{\infty} H_{n}^{\prime}(x, \xi) \sin v y \sin v \eta$ converges uniformly, and can be accurately computed by a direct truncation. The above estimate helps to find an approximate value of the truncating parameter $N$ required to attain a desired accuracy. 
Consider secondly the convergence of $\sum_{n=1}^{\infty} M_{n}(x, \xi) \sin v y \sin v \eta$, and the analysis of the modulus of its remainder,

$$
\begin{aligned}
\left|R_{N}(x, \mathrm{y} ; \xi, \eta)\right| & =\left|\sum_{n=N+1}^{\infty}\left[\frac{\exp (h(x+\xi-2 a))}{2 h}-\frac{\exp (v(x+\xi-2 a))}{2 v}\right] \sin v y \sin v \eta\right| \\
\leq & \left|\sum_{n=N+1}^{\infty}\left[\frac{\exp (h(x+\xi-2 a))}{2 h}-\frac{\exp (v(x+\xi-2 a))}{2 v}\right]\right|
\end{aligned}
$$

The inequality regarding the truncation error is obtained as follows

$$
\left|R_{N}(x, \mathrm{y} ; \xi, \eta)\right|<\frac{b}{\pi}\left\{[1-\exp (k(x-\xi))] \sum_{n=N+1}^{\infty} \frac{1}{n} \exp \left(\frac{n \pi}{b}(x-\xi)\right)+k \frac{b}{\pi} \sum_{n=N+1}^{\infty} \frac{1}{n^{2}}\right\} \text {. }
$$

Evidently,

$$
\sum_{n=N+1}^{\infty} \frac{1}{n^{2}}<\int_{N+1}^{\infty} \frac{1}{x^{2}} d x=\frac{1}{N+1}
$$

and

$$
\ln (1+x)<x, x<1
$$

Inequalities (A13)-(A15) allow estimation of the truncation error term:

$$
\begin{gathered}
\left|R_{N}(x, \mathrm{y} ; \xi, \eta)\right|<\frac{b}{\pi}\left\{[1-\exp (k(x-\xi))] \sum_{n=N+1}^{\infty} \frac{1}{n} \exp \left(\frac{n \pi}{b}(x-\xi)\right)+k \frac{b}{\pi} \sum_{n=N+1}^{\infty} \frac{1}{n^{2}}\right\} \\
<\frac{b}{\pi}\left\{[1-\exp (k(x-\xi))] \ln \left[1+\exp \left(\frac{\pi}{b}(x-\xi)\right)\right] \exp \left(\frac{N \pi}{b}(x-\xi)\right)+\frac{k b}{(N+1) \pi}\right\} \\
<\frac{b}{\pi}\left\{[1-\exp (k(x-\xi))] \exp \left(\frac{(N+1) \pi}{b}(x-\xi)\right)+\frac{k b}{(N+1) \pi}\right\} .
\end{gathered}
$$

Obviously, the above inequality is dependent on the variables. To prove the uniform convergence and obtain a uniform estimation requires further estimation of the right side of the above inequality. Therefore, consider the extremum property of the function

$$
\psi(\theta)=[1-\exp (k \theta)] \exp \left(\frac{(N+1) \pi \theta}{b}\right),(\theta<0) .
$$

It is not difficult to find that the stationary point is $\theta=\frac{1}{k} \ln \left(\frac{(N+1) \pi / b}{(N+1) \pi / b+k}\right)$ and the corresponding maximum is

$$
\psi_{\max }=\frac{k b /(N+1) \pi}{1+k b /(N+1) \pi} \exp \left[-\frac{(N+1) \pi}{b} \frac{1}{k} \ln \left(1-\frac{k b}{(N+1) \pi+k b}\right)\right] .
$$

When $N$ is sufficiently large, we have

$$
\begin{gathered}
\psi_{\max }<\frac{k b /(N+1) \pi}{1+k b /(N+1) \pi} \exp \left[-\frac{(N+1) \pi}{b} \frac{1}{k}\left(\frac{k b}{(N+1) \pi+k b}-\left(\frac{k b}{(N+1) \pi+k b}\right)^{2} / 2\right)\right] \\
<\frac{k b /(N+1) \pi}{1+k b /(N+1) \pi} \exp \left[-1+\frac{k b}{2(N+1) \pi+2 k b}\right]<\frac{k b}{(N+1) \pi} .
\end{gathered}
$$

Substituting the above inequality into inequality (A13) gives

$$
\left|R_{N}(x, y ; \xi, \eta)\right|<\frac{b}{\pi}\left[\frac{k b}{(N+1) \pi}+\frac{k b}{(N+1) \pi}\right]=\frac{2 k b^{2}}{(N+1) \pi^{2}} .
$$

Hence, the truncation error term converges to zero, and the function $\sum_{n=1}^{\infty} M_{n}(x, \xi) \sin v y \sin v \eta$ converges uniformly. The resulting uniform estimator of function $\sum_{n=1}^{\infty} M_{n}(x, \xi) \sin v y \sin v \eta$ is advantageous to the approximate calculation. 
Then we find that the two series in Equation (A10) converge uniformly and can be accurately computed by a direct truncation. The above estimation can provide an appropriate value of the truncating parameter $N$ required to attain a desired accuracy.

$$
\begin{aligned}
G(x, y ; \xi, \eta)=\frac{2}{b} \sum_{n=1}^{\infty} g_{n}(x, \xi) \sin v y \sin v \eta \\
=\frac{1}{4 \pi}\left[\ln \frac{1-2 \exp (c(x+\xi-2 a)) \cos (y+\eta)+\exp (2 c(x+\xi-2 a))}{1-2 \exp (c(x+\xi-2 a)) \cos (y-\eta)+\exp (2 c(x+\xi-2 a))}\right. \\
\quad+\ln \frac{1-2 \exp (c(x-\xi)) \cos (y+\eta)+\exp (2 c(x-\xi))}{1-2 \exp (c(x-\xi)) \cos (y-\eta)+\exp (2 c(x-\xi))} \\
\left.\quad-\ln \frac{1-2 \exp (-c(x+\xi)) \cos (y+\eta)+\exp (-2 c(x+\xi))}{1-2 \exp (-c(x+\xi)) \cos (y-\eta)+\exp (-2 c(x+\xi))}\right] \\
+\frac{2}{b} \sum_{n=1}^{N} H_{n}^{\prime}(x, \xi) \sin v y \sin v \eta+\frac{2}{b} \sum_{n=1}^{N} M_{n}(x, \xi) \sin v y \sin v \eta+O\left(\frac{1}{N}\right)
\end{aligned}
$$

Similarly, interchanging the variables $x$ with $\xi$ in the first series gives the representation of $G(x, y ; \xi, \eta)$ valid for $(x \geq \xi)$.

\section{References}

1. Talagrand, O.; Courtier, P. Variational assimilation of meteorological observations with the adjoint vorticity equation. I: Theory. Q. J. R. Meteorol. Soc. 1987, 113, 1311-1328. [CrossRef]

2. Al-Jamal, M.F. Numerical Solution of Elliptic Inverse Problems via the Equation Error Method. Ph.D. Thesis, Michigan Technological University, Houghton, MI, USA, 2012.

3. Mehraliyev, Y.T.; Kanca, F. An inverse boundary value problem for a second order elliptic equation in a rectangle. Math. Modeling Anal. 2014, 19, 241-256. [CrossRef]

4. Mehraliyev, Y.T. On solvability of an inverse boundary value problem for a fourth order elliptic equation. J. Math. Syst. Sci. 2013, 3, 560-566.

5. Orlovsky, D.G. Inverse problem for elliptic equation in Banach space with Bitsadze-Samarsky boundary value conditions. J. Inverse ILL-Posed Probl. 2013, 21, 141-157. [CrossRef]

6. Ming, W.; Renqing, D.; Wenzhong, G.; Takeda, T. Retrieval Single-Doppler Radar Wind with Variational Assimilation MethodPart I: Objective Selection of Functional Weighting Factors. Adv. Atmos. Sci. 1998, 15, 553-567. [CrossRef]

7. Ming, W.; Guo-qing, L.; Cheng-gang, W.; Wen-zhong, G.; Qin, X. Optimal Selection for the Weighted Coefficients of the Constrained Variational Problems. Appl. Math. Mech. 2003, 24, 926-944. [CrossRef]

8. Melnikov, Y.A. Construction of Green's functions for the two-dimensional static Klein-Gordon equation. J. Part. Differ. Equ. 2011, 24, 114-139.

9. Aseeri, S.; Batrašev, O.; Icardi, M.; Leu, B.; Liu, A.; Li, N.; Muite, B.K.; Müller, E.; Palen, B.; Quell, M. Solving the Klein-Gordon equation using Fourier spectral methods: A benchmark test for computer performance. In Proceedings of the Symposium on High Performance Computing (HPC'15), Montreal, QC, Canada, 17 June 2015; pp. 182-191.

10. Gravel, P.; Cauthier, C. Classical applications of the Klein-Gordon equation. Am. J. Phys. Teach. 2011, 79, 447-453. [CrossRef]

11. Muravey, D. The boundary value problem for a static 2D Klein-Gordon equation in the infinite strip and in the half-plane. Mathematics 2015, 40, 205-227.

12. Cheng, H.; Mu, X.; Jiang, H.; Liu, G.; Wei, M. Green's Function for the boundary value problem of thestatic Klein-Gordon equation stated on a rectangular region and its convergence analysis. Bound. Value Probl. 2017, 2017, 72. [CrossRef]

13. Courant, R.; Hilbert, D. Methods of Mathematical Physics; Interscience Publishers Inc.: New York, NY, USA, 1953.

14. Sasaki, Y.K. Some basic formulisms in numerical variational analysis. Mon. Weather Rev. 1970, 98, 875-883. [CrossRef] 